\title{
Validation of a newly proposed histopathological classification in Japanese patients with anti-neutrophil cytoplasmic antibody-associated glomerulonephritis
}

Takashi Iwakiri ${ }^{1}$, Shouichi Fujimoto ${ }^{2 *}$, Kiyoki Kitagawa ${ }^{3}$, Kengo Furuichi ${ }^{3}$, Junya Yamahana ${ }^{4}$, Yunosuke Matsuura ${ }^{1}$, Atsushi Yamashita ${ }^{1}$, Shigehiro Uezono ${ }^{5}$, Yoshiya Shimao $^{6}$, Shuichi Hisanaga ${ }^{7}$,Takeshi Tokura ${ }^{8}$, Takashi Wada ${ }^{9}$, Kazuo Kitamura ${ }^{10}$ and Yujiro Asada ${ }^{1}$

\begin{abstract}
Background: A new histopathological classification of anti-neutrophil cytoplasmic antibody (ANCA)-associated glomerulonephritis was recently proposed. We evaluated the predictive value of this classification for renal outcome in Japanese patients.

Methods: We enrolled 122 patients with ANCA-associated glomerulonephritis diagnosed at several institutions in Japan between January 2000 and March 2010. Twenty patients were excluded because of observation durations of $<1$ year, and/or because their biopsy specimens contained $<10$ glomeruli. Renal biopsy specimens were categorized into four classes according to the proposed classification. We evaluated the predictive value of immunohistochemical staining for a-smooth muscle actin (SMA), Wilm's tumor 1 (WT1), CD68, and cytokeratin for end-stage renal disease (ESRD).
\end{abstract}

Results: The study population included 54 men and 48 women. Age, estimated glomerular filtration rate (eGFR), and proteinuria were $66.3 \pm 11.3$ years, $21.6 \mathrm{ml} / \mathrm{min}$. and $1.10 \mathrm{~g} / 24 \mathrm{~h}$, respectively. Eighty-six patients were positive for myeloperoxidase-ANCA, five were positive for proteinase 3-ANCA, and 11 were negative for both antibodies. Median follow-up time was 41.0 months. Twenty-three patients (22.5\%) developed ESRD during the follow-up period. Twelve patients died during follow up; 7/12 patients developed ESRD before death, and 5/12 patients died without ESRD. The incidence of ESRD increased with sequential categories: focal, 2/46 (4.3\%); crescentic, 9/32 (28\%); mixed, 8/18 (44\%); and sclerotic, $4 / 6$ (67\%). The focal class had the best renal survival and the sclerotic class had the worst renal survival $(p<0.001)$. Kaplan-Meier renal survival analysis was similar to that of the new classification system proposal. In the multivariate analysis, the classification system tended to be a prognostic factor for $\operatorname{ESRD~}(p=0.0686$, crescentic, mixed and sclerotic vs. focal, hazard ratio (HR) [95\% confidence interval, Cl]; 2.99 [0.61-22.7], 5.04 [1.11-36.4] and 9.93 [1.53-85.7], respectively). a-SMA-positivity also tended to be associated with $\operatorname{ESRD}(p=0.1074)$.

Conclusion: The new histopathological classification was associated with eGFR at 1 year and tended to be associated with ESRD in our Japanese cohort with ANCA-associated glomerulonephritis. a-SMA positivity might be an additional prognostic factor for ESRD.

Keywords: Anti-neutrophil cytoplasmic antibody, Histopathological classification, Immunohistochemistry, a-Smooth muscle actin

\footnotetext{
* Correspondence: fujimos@fc.miyazaki-u.ac.jp

${ }^{2}$ Department of Hemovascular Medicine and Artificial Organs, University of

Miyazaki, 5200 Kihara, Kiyotake, Miyazaki 889-1692, Japan

Full list of author information is available at the end of the article
} 


\section{Background}

Anti-neutrophil cytoplasmic antibody (ANCA)-associated glomerulonephritis is a common cause of rapidly progressive glomerulonephritis in adults [1]. Three major categories have been defined: microscopic polyangiitis (MPA), granulomatosis with polyangiitis (GPA), and eosinophilic granulomatosis with polyangiitis (EGPA) [2,3]. The histopathological findings are characterized by various lesions, particularly extracapillary proliferation and fibrinoid necrosis; however, lesions such as fibrous crescent formation and focal or global glomerular sclerosis, indicating chronic lesions, are also observed [4]. The renal prognosis of ANCA-associated glomerulonephritis differs greatly between individual patients. Several studies have determined the clinical and histopathological predictors of renal outcome, and shown that low levels of serum creatinine ( $\mathrm{SCr}$ ) at diagnosis and a high percentage of normal glomeruli were predictors for a better renal outcome, whereas a high percentage of sclerotic glomeruli was a predictor for a worse renal outcome [5-8]. However, a standardized histopathological classification of this disease has been lacking for many years.

A histopathological classification of ANCA-associated glomerulonephritis was recently proposed, based on an analysis of 100 patients from multiple centers in Europe [9]. This classification system is based on glomerular pathology and defines four classes: focal, crescentic, mixed and sclerotic. The authors reported that the phenotypic order of the class corresponded to progressive worsening of renal function. Japanese patients with ANCA-associated vasculitis show a different distribution of ANCA subtypes compared with patients from Western countries, with higher rates of myeloperoxidase (MPO)-ANCA expression than proteinase 3 (PR3)-ANCA $[10,11]$. We therefore performed a validation study to determine whether this proposed histopathological classification could be applied to Japanese patients with ANCAassociated glomerulonephritis.

\section{Methods}

\section{Patients}

We enrolled 122 patients diagnosed with biopsy-confirmed ANCA-associated glomerulonephritis between January 2000 and March 2010 from six institutions in Japan (Miyazaki University, Kanazawa University, Toyama Prefectural Central Hospital, Miyazaki Prefectural Hospital, Koga General Hospital and Miyazaki Social Insurance Konan Hospital). Patients with primary renal vasculitis were defined in accordance with the following criteria: new patients with MPA, GPA, EGPA or renal limited vasculitis (RLV) and renal involvement (elevated $\mathrm{SCr}$, hematuria, proteinuria, or red cell casts) attributable to active vasculitis with or without other organ involvement, and positive serology for ANCA. Histological confirmation of renal involvement was the finding of necrotizing vasculitis and pauci-immune crescentic glomerulonephritis in the renal biopsy. The European Medicines Agency (EMEA) algorithm [12] was used to define MPA, GPA and EGPA. This algorithm utilizes the American College of Rheumatology criteria (1990) and the Chapel Hill Consensus Conference definitions. Using this approach, RLV is placed within MPA. ANCA-negative patients were eligible for enrollment in this study if there was histological confirmation of renal involvement. Patients who were followed up for $<1$ year $(n=14)$ or whose biopsy specimens contained $<10$ glomeruli $(n=6)$ were excluded. A total of 102 patients were therefore included in this study. Most of the patients received intravenous and/or oral methylprednisolone therapy with or without immunosuppressants after diagnosis, according to the attending physician's judgement. The ethical committee of University of Miyazaki approved this study (approval number: 2010-751).

\section{Treatment}

In this present study, $83(81.4 \%)$ cases received methylprednisolone pulse therapy, and $89(87.3 \%)$ cases were administered oral prednisolone. Furthermore, 30 (29.4\%) cases were treated with cyclophosphamide (CYC): 15 (14.7\%) were treated with intravenous CYC pulse therapy (IV-CY), 11 (10.8\%) with oral CYC therapy, and four (3.9\%) with both IV-CY and oral CYC. Twenty-one (20.6\%) cases were treated with mizoribine (MZB), three $(2.9 \%)$ cases were treated with azathioprine (AZA), and one $(1.0 \%)$ case was treated with gusperimus hydrochloride.

\section{Patient parameters and outcomes}

This was a retrospective study based on clinical information obtained from hospital records and general practice. $\mathrm{SCr}$ and the degree of proteinuria were measured at the time of renal biopsy, prior to the initiation of immunosuppressive therapy or hemodialysis. SCr was evaluated every 1-3 months after the initiation of therapy. The development of ESRD during follow-up was the primary outcome. ESRD was defined as requiring permanent renal replacement therapy. Estimated glomerular filtration rate (eGFR) at 1 year was the secondary outcome.

\section{Calculation of eGFR}

SCr was assayed by an enzymatic method and eGFR was calculated using the following equation [13]: eGFR (ml/ $\mathrm{min} / 1.73 \mathrm{~m}^{2}$ ) $=194 \times$ age $(\text { years })^{-0.287} \times$ serum creatinine $(\mathrm{mg} / \mathrm{dl})^{-1.094}(\times 0.739$ for women $)$.

\section{Renal histopathology}

Renal biopsy specimens were examined by light microscopy and immunofluorescence analysis. For light microscopy, tissues were stained with hematoxylin and eosin, 
periodic acid-Schiff (PAS), AZAN, and methenamine silver. The median number of glomeruli per biopsy was 19.0 (range 10-61). For immunofluorescence analysis, sections were incubated with antibodies against IgG, IgA, IgM, C3, C4, C1q, and fibrinogen, to exclude other renal diseases. Renal biopsies were categorized into four classes in accordance with the newly proposed histopathological classification. Samples with $\geq 50 \%$ normal glomeruli were classified as focal, those with $\geq 50 \%$ cellular crescentic glomeruli were classified as crescentic, and those with $\geq 50 \%$ globally sclerotic glomeruli were classified as sclerotic. The other cases, those with $<50 \%$ normal, cellular crescentic, and globally sclerotic glomeruli were classified as mixed. Two independent nephropathologists (T.I. and K.K.) who were blinded to the patients' characteristics performed the histopathological evaluations and classified the specimens into the four classes. Inter-observer variation of categorization according to the new histopathological classification was tested $(\kappa=$ 0.91). Discrepancies between the observers were resolved by conference to achieve consensus.

\section{Immunohistochemical analysis}

Immunohistochemical staining was performed on samples from 34 patients with crescentic or mixed class for $\alpha$-smooth muscle actin (SMA; myoepithelial marker; clone: 1A4, mouse monoclonal antibody; Dako, Glostrup, Denmark), Wilm's tumor 1 (WT1; podocyte marker; clone: 6F-H2, mouse monoclonal antibody; Dako), CD68 (macrophage marker; clone: PG-M1, mouse monoclonal antibody; Dako) and cytokeratin (epithelial marker; clone: AE1/AE3, mouse monoclonal antibody; Dako), using 4- $\mu$ m-thick sections of formalin-fixed and paraffin-embedded tissue. Sections were subsequently stained with Envision (Dako). Horseradish peroxidase activity was visualized using 3,3'diaminobenzidine tetrahydrochloride, and counterstaining with Meyer's hematoxylin. Of the 50 cases in either the crescentic or mixed class, 16 cases did not have sufficient tissue remaining for immunohistochemical analysis.

Immunoreactivity was evaluated by two investigators unaware of the patients' characteristics (T.I. and A.Y.). A cell was considered positive for $\alpha$-SMA, CD68 and/or cytokeratin in case of cytoplasmic staining. A cell was considered positive for WT1 in case of nuclear staining. We assessed normal glomeruli in each sample and counted all immunopositive cells. The definition of a normal glomerulus was a glomerulus without collapse, crescent formation, adhesion, or focal to segmental/global glomerulosclerosis. Even if the $\alpha$-SMA-positive area was very small, the glomerulus was considered to be immunopositive. Reproducibility was assessed by blinded replicate counting of immunopositive cells performed by the two observers; the inter-observer correlation coefficients for $\alpha$-SMA, WT1, CD68 and cytokeratin were $r=0.86,0.77,0.91,0.87$, respectively. Discrepancies between the observers were resolved by conference to achieve consensus.

\section{Statistical analysis}

Data were analyzed using the Mann-Whitney test, Fisher's exact test, and Kruskal-Wallis test and post hoc analysis (Dunn's multiple comparison test) as appropriate. KaplanMeier survival analysis was used to compare renal survival. Multivariate analysis of renal survival was performed by Cox regression analysis. Patients who died before developing ESRD were treated as censored in Kaplan-Meier renal survival analysis and Cox regression analysis. Values of $p<0.05$ were considered to be statistically significant. JMP 8.0.1 (SAS, Cary, NC, USA) and GraphPad Prism 5.01 (GraphPad Software, San Diego, CA, USA) were used for statistical analyses.

\section{Results}

\section{Clinical and histopathological studies}

Overall, 23 patients developed ESRD that required maintenance hemodialysis therapy during follow-up [median, 41.0 months, interquartile range (IQR), 20.0-63.8]. The baseline characteristics at the time of diagnosis for patients with and without ESRD are shown in Table 1. The mean age ( \pm standard deviation) was $66.3 \pm 11.3$ years. There were 54 males and 48 females. All patients underwent serologic tests for MPO-ANCA and PR3-ANCA using enzyme-linked immunosorbent assays. Eighty-six patients were positive for MPO-ANCA and five patients were positive for PR3-ANCA; none were positive for both antibodies. Although 11 patients were negative for both antibodies, their disease was confirmed by typical histopathological features and clinical findings. The median $\mathrm{SCr}$ measured at the time of renal biopsy before starting immunosuppressive therapy or hemodialysis was $1.95 \mathrm{mg} / \mathrm{dl}$ (IQR, 1.30-4.15) and the median eGFR was $21.6 \mathrm{ml} / \mathrm{min} / 1.73 \mathrm{~m}^{2}$ (IQR, 11.5-39.5). The median proteinuria was $1.10 \mathrm{~g} / 24 \mathrm{~h}$ (IQR, 0.35-2.12) based on 24-h urine collection. Age, sex, serum albumin and serum C-reactive protein (CRP) were not significantly different between patients with or without ESRD. However, eGFR and 24-h proteinuria at diagnosis did differ significantly between patients with and without ESRD [eGFR: 25.7 versus (vs.) $9.0 \mathrm{ml} / \mathrm{min} / 1.73 \mathrm{~m}^{2}, p<0.001$; proteinuria: 1.8 vs. $0.7 \mathrm{~g} / 24 \mathrm{~h}, p<0.01]$. According to the EMEA algorithm, 97 cases were classified as MPA, three as GPA and two as EGPA. Systemic organ involvements were as follow: ear, nose and throat, six patients (5.9\%); respiratory system, 20 patients (19.6\%); and nervous system, 12 patients (11.8\%). The 102 patients were then categorized into four classes according to the newly proposed histopathological classification: 46 patients with focal, 32 patients with crescentic, 18 patients with mixed, and six patients with sclerotic class. The clinical and histopathological findings in the four 
Table 1 Patient characteristics at the time of diagnosis

\begin{tabular}{|c|c|c|c|c|}
\hline & Total $(n=102)$ & Non-ESRD $(n=79)$ & ESRD during follow-up $(n=23)$ & $p$ value \\
\hline Age (years) & $66.3 \pm 11.3$ & $66.3 \pm 12.2$ & $66.3 \pm 7.7$ & n.s. \\
\hline Male/female, $n$ & $54 / 48$ & $39 / 40$ & $15 / 8$ & n.s. \\
\hline MPO-ANCA, $n$ & 86 & 70 & 16 & \\
\hline PR3-ANCA, $n$ & 5 & 4 & 1 & \\
\hline Both negative, $n$ & 11 & 5 & 6 & \\
\hline $\mathrm{eGFR}\left(\mathrm{ml} / \mathrm{min} / 1.73 \mathrm{~m}^{2}\right)$ & $21.6(11.5-39.5)$ & $25.7(17.4-50.4)$ & $9.0(5.1-13.3)$ & $<0.001$ \\
\hline Serum albumin (g/dl) & $3.1(2.7-3.7)$ & $3.1(2.7-3.7)$ & $3.1(2.7-3.8)$ & n.s. \\
\hline Serum CRP (mg/dl) & $3.35(0.98-8.98)$ & $3.70(1.08-10.0)$ & $2.13(0.20-6.70)$ & n.s. \\
\hline Proteinuria (g/day) & $1.10(0.35-2.12)$ & $0.70(0.30-1.77)$ & $1.80(1.10-2.50)$ & $<0.01$ \\
\hline
\end{tabular}

Data are expressed as mean \pm SD or median (interquartile range). $p$ values were calculated using the Mann-Whitney $U$ test or Fisher's exact test. Statistical significance was defined as $p<0.05$.

Abbreviations: ESRD end-stage renal disease, ns not significant, eGFR estimated glomerular filtration rate, MPO myeloperoxidase, PR3 proteinase-3, CRP C-reactive protein.

histopathological classes are shown in Table 2. There were no significant differences in age or gender among the four classes. PR3-ANCA-positive patients occurred in the focal class $(n=3)$ and the crescentic class $(n=2)$, but not in the mixed or sclerotic class. Serum CRP was higher in the focal and crescentic class than in the mixed and sclerotic class. Proteinuria was lower in the focal class than in the other three classes. The proportion of normal glomeruli was higher in the mixed class than in the crescentic class, but the proportion of sclerotic glomeruli was also higher in the mixed class than in the crescentic class. At the time of diagnosis, the median eGFRs in the focal, crescentic, mixed, and sclerotic class were 38.1, 12.0, 16.5, and $12.4 \mathrm{ml} / \mathrm{min} / 1.73 \mathrm{~m}^{2}$, respectively. eGFR was thus significantly different in the focal class compared with the other classes $(p<0.001$ vs. crescentic; $p<0.01$ vs. mixed; $p<0.05$ vs. sclerotic). However, there were no significant differences among the crescentic, mixed, and sclerotic class. One year after the initial diagnosis, the median eGFRs were $45.7,24.5,26.0$, and $16.9 \mathrm{ml} / \mathrm{min} / 1.73 \mathrm{~m}^{2}$ in the focal, crescentic, mixed, and sclerotic class, respectively. Except for patients who developed ESRD, eGFR at 1 year showed an improvement from that at diagnosis in all four classes. Again, eGFR differed significantly between

Table 2 Baseline characteristics of patients in each histopathological class

\begin{tabular}{|c|c|c|c|c|c|}
\hline & Focal & Crescentic & Mixed & Sclerotic & $p$ value \\
\hline Age (years) & $66.1 \pm 11.4$ & $66.3 \pm 12.5$ & $66.7 \pm 10.2$ & $67.5 \pm 8.6$ & n.s. \\
\hline Male/female, $n$ & $24 / 22$ & $15 / 17$ & $10 / 8$ & $5 / 1$ & n.s. \\
\hline MPO-ANCA, $n$ & 40 & 26 & 15 & 5 & n.s. \\
\hline PR3-ANCA, $n$ & 3 & 2 & 0 & 0 & \\
\hline Both negative, $n$ & 3 & 4 & 3 & 1 & \\
\hline eGFR (ml/min/1.73 m²) & $38.1(22.5-57.4)$ & $12.0(7.1-19.7)$ & $16.5(8.7-31.6)$ & $12.4(9.8-27.4)$ & $<0.0001$ \\
\hline Serum albumin (g/dl) & $3.4(2.8-3.7)$ & $2.7(2.4-3.3)$ & $3.1(2.8-3.7)$ & $3.8(3.5-3.9)$ & $<0.01$ \\
\hline Serum CRP (mg/dl) & $4.13(1.43-9.62)$ & $4.98(1.65-14.0)$ & $1.21(0.10-3.93)$ & $0.16(0.10-1.10)$ & $<0.001$ \\
\hline Proteinuria (g/day) & $0.35(0.21-0.79)$ & $1.50(1.04-2.50)$ & $1.75(1.07-2.20)$ & $1.50(0.25-3.98)$ & $<0.0001$ \\
\hline Immunosuppressants, yes/no, $n$ & $25 / 21$ & $17 / 15$ & $4 / 14$ & $1 / 5$ & $<0.05$ \\
\hline \multicolumn{6}{|l|}{ Histopathological findings } \\
\hline Normal glomeruli (\%) & $73.2(60.0-81.6)$ & $17.9(10.6-28.0)$ & $35.4(22.5-43.8)$ & $11.3(0-22.0)$ & $<0.0001$ \\
\hline Crescentic glomeruli (\%) & $13.8(8.9-23.6)$ & $67.7(60.0-74.7)$ & $31.4(15.6-40.4)$ & $12.4(3.3-22.4)$ & $<0.0001$ \\
\hline Globally sclerotic glomeruli (\%) & $6.5(0-13.4)$ & $3.2(0-13.1)$ & $29.3(19.0-40.4)$ & $64.5(54.9-71.1)$ & $<0.0001$ \\
\hline
\end{tabular}

Data are expressed as mean \pm SD or median (interquartile range).

Abbreviations: $n s$ not significant, GFR estimated glomerular filtration rate, MPO myeloperoxidase, $P R 3$ proteinase-3, CRP C-reactive protein Immunosuppressants were administered after the diagnosis of ANCA-associated glomerulonephritis.

eGFR: focal vs. crescentic***, focal vs. mixed**, focal vs. sclerotic*, Serum albumin: focal vs. crescentic*, crescentic vs. sclerotic**, Serum CRP: focal vs. mixed*, focal vs. sclerotic*, crescentic vs. mixed ${ }^{*}$, crescentic vs. sclerotic**, Proteinuria: focal vs. crescentic ${ }^{* *}$, focal vs. mixed ${ }^{* *}$, Immunosuppressants: focal vs. mixed ${ }^{*}$, crescentic vs. mixed ${ }^{*}$, Normal glomeruli: focal vs. crescentic***, focal vs. mixed***, focal vs. sclerotic***, Sclerotic glomeruli: focal vs. crescentic***, crescentic vs. mixed***, crescentic vs. sclerotic***, Globally sclerotic glomeruli: focal vs. mixed ${ }^{* * *}$, focal vs. sclerotic ${ }^{* * *}$, crescentic vs. mixed ${ }^{* * *}$, crescentic vs. sclerotic ${ }^{* * *},{ }^{*} p<0.05$, ${ }^{* *} p<0.01,{ }^{* * *} p<0.001$. 
the focal class and the other classes $(p<0.001$ vs. crescentic; $p<0.05$ vs. mixed; $p<0.01$ vs. sclerotic), but there were no significant differences among the crescentic, mixed and sclerotic class (Figure 1).

At 1 year after initial diagnosis, $\mathrm{SCr}$ had doubled in two patients, and another 10 patients had developed ESRD. The incidences of these events combined at 1 year were $2.2 \%$ (1/46), $21.9 \%$ (7/32), $11.1 \%(2 / 18)$, and $33.3 \%(2 / 6)$ in the focal, crescentic, mixed, and sclerotic class, respectively. During the entire follow-up period, 23 patients (22.5\%) developed ESRD and 12 patients died. Seven of the 12 patients who died developed ESRD before death, and the remaining five died without ESRD. The proportions of patients who developed ESRD during follow up increased with sequential category, [focal, 4.3\% (2/46); crescentic, $28.1 \%$ (9/32); mixed, $44.4 \%$ (8/18); sclerotic, $66.6 \%$ (4/6); Table 3]. Kaplan-Meier analysis of renal survival showed that prognosis was best for patients in the focal class and worst for patients in the sclerotic class $(p<0.001)$. Kaplan-Meier analysis found no significant difference between the crescentic and mixed class $(p=0.2716)$. However, the Kaplan-Meier renal survival curve was almost similar to the curve of Berden et al. [9] (Figure 2). In the multivariate Cox regression analysis, the new classification system at diagnosis tended to be a prognostic factor for ESRD during the follow-up period $(p=0.0686$, crescentic, mixed and sclerotic vs. focal, hazard ratio (HR) [95\% confidence interval, CI]; 2.99 [0.6122.7], 5.04 [1.11-36.4] and 9.93 [1.53-85.7], respectively). Additionally, eGFR at diagnosis also tended to be a prognostic factor for ESRD during the follow-up period ( $p=0.0619$, HR [95\% CI]; 0.97 [0.93-1.00]) (Table 4). Analysis of the incidence of ESRD according to treatment did not identify immunosuppressant use as a prognostic factor for ESRD $(p=0.8390, \mathrm{HR}[95 \% \mathrm{CI}]$; 0.90 [0.31-2.45]). In addition, there was no significant difference between patients treated with or without CYC (data not shown).
Table 3 Proportions of ESRD according to the histopathological classes

\begin{tabular}{lll}
\hline Class & $\mathbf{1}$ year after diagnosis & Total follow-up period \\
\hline Focal & $1 / 46(2.2 \%)$ & $2 / 46(4.3 \%)$ \\
Crescentic & $7 / 32(21.9 \%)$ & $9 / 32(28.1 \%)$ \\
Mixed & $2 / 18(11.1 \%)$ & $8 / 18(44.4 \%)$ \\
Sclerotic & $2 / 6(33.3 \%)$ & $4 / 6(66.6 \%)$ \\
\hline
\end{tabular}

\section{Immunohistochemical study}

Because histopathological evaluation by light microscopy could not differentiate the crescentic and mixed class in terms of ESRD, we evaluated the ability of immunohistochemical studies to predict renal outcome. We performed immunohistochemical staining for $\alpha$-SMA, WT1, CD68, and cytokeratin on samples from 34 patients with crescentic or mixed class. There were no significant differences in expression levels of WT1, CD68, and cytokeratin between patients with and without ESRD (data not shown). By contrast, $\alpha$-SMA immunoreactivity differed substantially between each normal glomerulus. As shown in Figure 3A, there was no immunoreactivity for $\alpha$-SMA in this normal glomerulus, except in the glomerular vascular pole and surrounding Bowman's capsule. On the other hand, Figure 3B shows marked immunoreactivity for $\alpha$-SMA in a normal glomerulus. We counted the number of normal glomeruli in each case and then assessed if they were immunopositive for $\alpha$-SMA or not. The mean proportion of $\alpha$-SMA-positive glomeruli per normal glomeruli was $82.9 \%$. Receiver operating characteristics analysis was used to identify the optimal cut-off rate of $\alpha$-SMA-positive glomeruli per normal glomeruli for distinguishing renal outcome. A cut-off score of $83.3 \%$ optimally identified $100 \%$ of ESRD cases (sensitivity) and $46.7 \%$ of non-ESRD cases (specificity). In KaplanMeier analysis, higher $\alpha$-SMA expression tended to be associated with poorer renal survival (Figure 3C).
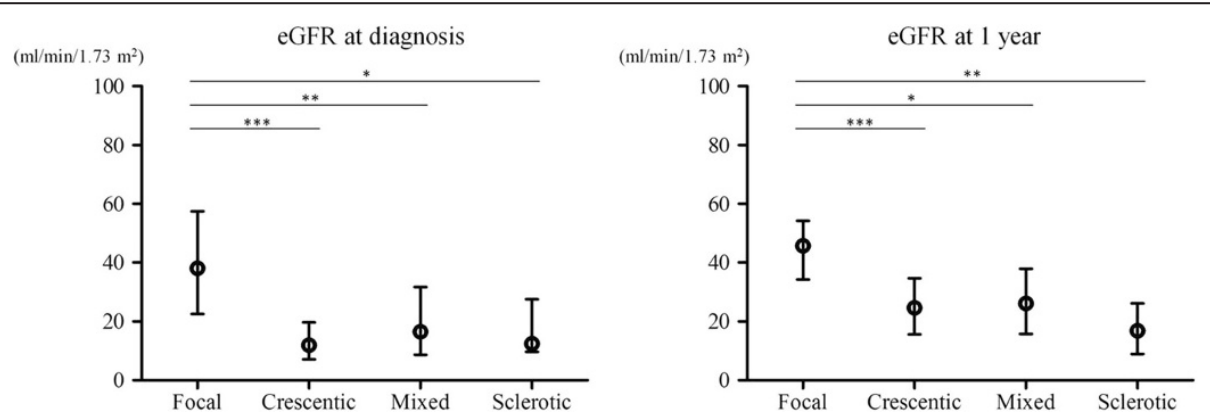

Figure 1 Association between histopathological class and eGFR. eGFRs at diagnosis and 1 year after diagnosis were higher in the focal class than in the other classes. There were no significant differences in eGFR among the crescentic, mixed, and sclerotic class. Abbreviations: eGFR, estimated glomerular filtration rate; blank circles, median values; error bars, interquartile ranges. ${ }^{*} p<0.05$, ${ }^{* *} p<0.01$, and ${ }^{* * *} p<0.001$ (post hoc analysis). 


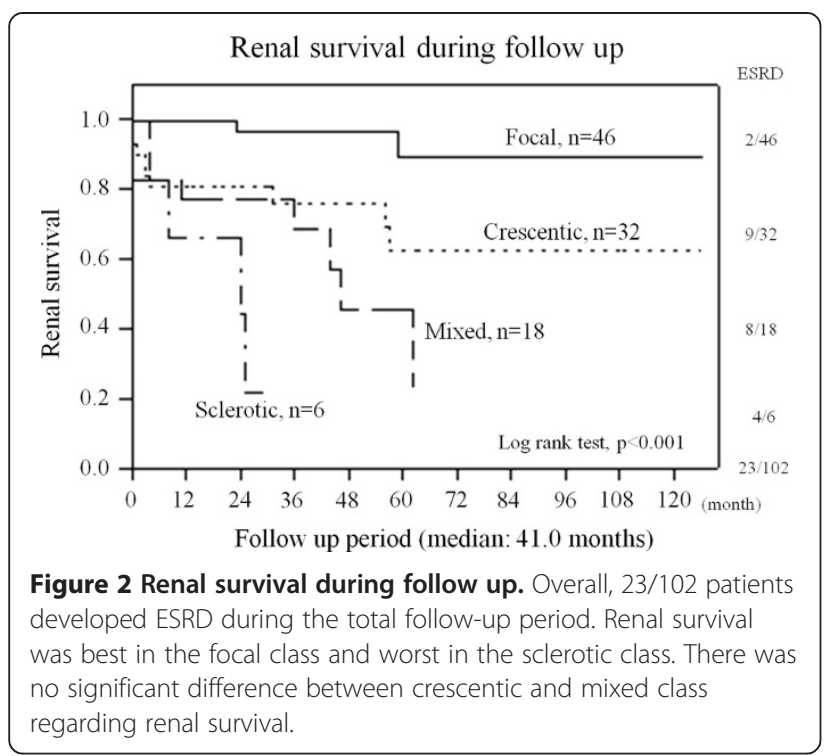

\section{Discussion}

\section{Clinical and histopathological findings}

Several attempts have been made to establish histopathological criteria for ANCA-associated glomerulonephritis and to predict ESRD and overall survival in this disease $[14,15]$. However, studies investigating the clinical and histopathological predictors of renal outcome have provided different results with some overlap, in terms of the predictive values of normal glomeruli, glomerulosclerosis and eGFR at diagnosis $[4,5,16-19]$. Most of the proposed classification systems are cumbersome and require histopathological evaluation as well as clinical information. Berden et al. recently investigated the prognostic value of a simple histopathological classification, based on an analysis of 100 patients across multiple centers in Europe [9]. We examined the suitability of this classification system for Japanese patients with ANCA-associated glomerulonephritis. This histopathological classification was associated with eGFR at 1 year in our cohort, as in Berden et al.'s

Table 4 Multivariate analysis of progression to ESRD

\begin{tabular}{lll}
\hline Variable & $\boldsymbol{p}$ value & HR $(\mathbf{9 5 \%} \mathbf{C l})$ \\
\hline Age & 0.2336 & $1.03(0.98-1.09)$ \\
Male & 0.1072 & $2.33(0.84-7.08)$ \\
eGFR at diagnosis & 0.0619 & $0.97(0.93-1.00)$ \\
Proteinuria at diagnosis & 0.1059 & $1.23(0.95-1.54)$ \\
Immunosuppressant, yes/no & 0.8390 & $0.90(0.31-2.45)$ \\
Histopathological & 0.0686 & crescentic vs. focal, $2.99(0.61-22.7)$ \\
classification & & mixed vs. focal, 5.04 (1.11-36.4) \\
& & sclerotic vs. focal, 9.93 (1.53-85.7) \\
\hline
\end{tabular}

Abbreviations: ESRD end-stage renal disease, HR hazard ratio, $\mathrm{Cl}$ confidence interval. study. The incidence of ESRD during the follow-up period increased with sequential category of the proposed classification. Although the Kaplan-Meier survival curves showed the same distribution as the study by Berden et al., the histopathologic classification was not associated with ESRD during follow up in our cohort in the multivariate analysis. We think there are several possible explanations for why we did not find an association between the classification and developing ESRD. First, Berden et al.'s study was conducted in Europe, and of the 100 patients, 45 were positive for PR3-ANCA and 47 were positive for MPOANCA. Notably, the distribution of ANCA subtypes in Japanese patients with ANCA-associated vasculitis differs from that in Western countries. For example, Japanese patients are more frequently positive for MPO-ANCA than PR3-ANCA [10,11]. Indeed, our cohort consisted of 86 patients with MPO-ANCA-positive disease and just five with PR3-ANCA-positive disease, while the remaining 11 patients were negative for both antibodies. The different distributions of MPO- and PR3-ANCA between Europe and Japan may thus have contributed to the conflicting results. However, we were unable to conclude if the mechanism of glomerular injury was due to the different type of ANCA, though patients with MPO-ANCA do show more active and chronic lesions than PR3-ANCA patients [20]. In addition, differences in genetic backgrounds may affect the renal pathology findings and clinical courses in patients with ANCA vasculitis. International collaborative studies are clearly needed to determine the causes of the different phenotypes and clinical courses among different populations.

A second possible reason for the apparent discrepancy between the current results and those of Berden et al. [9] may be the difference in treatments used in Europe and Japan. In Europe, glucocorticoids in combination with CYC or methotrexate is the standard therapeutic strategy for ANCA-associated vasculitis [21], while in Japan, although glucocorticoids in combination with $\mathrm{CYC}$ is also recommended as a treatment for ANCA-associated vasculitis depending on the severity of disease, CYC is only used in $33.7-45.2 \%$ of Japanese patients with ANCA-associated systemic vasculitis [22]. CYC is used less frequently in Japan to avoid associated events of infection, though its low usage may also facilitate disease remission.

Age, sex, and eGFR at diagnosis have been reported to be important predictors of renal outcome [5-8,18,23]. However, the current study found no significant differences in these factors at diagnosis between the crescentic and mixed class. Other reports have confirmed the importance of normal glomeruli for better renal outcome $[6,8,18]$. In our series, the proportion of normal glomeruli was higher in the mixed class than in the crescentic class, but the proportion of sclerotic glomeruli was also higher in the mixed class than in the crescentic 

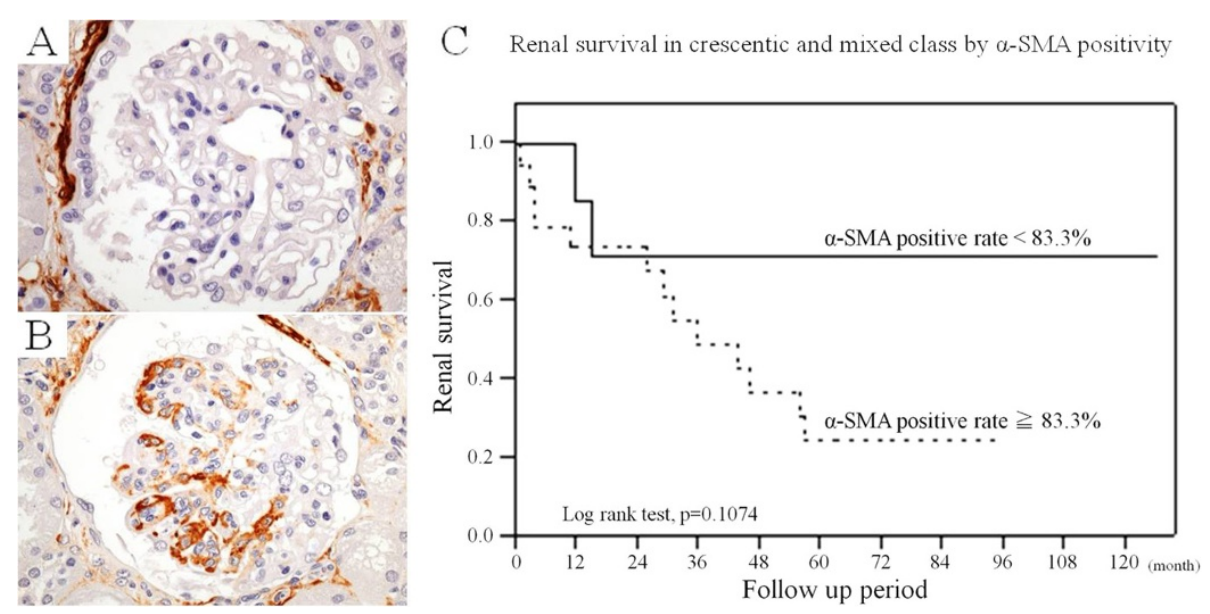

Figure 3 Comparison of renal survival according to a-SMA expression in crescentic and mixed classes. (A) Tissue section showing absence of a-SMA staining in a normal glomerulus, except in the glomerular vascular pole and thickened Bowman's capsule. (B) Tissue section showing marked a-SMA expression in a normal glomerulus, suggesting activation of a mesangial cell. (C) Higher a-SMA positivity tended to be associated with poor renal survival. Abbreviation: SMA, smooth muscle actin.

class. These features probably hindered discrimination between the crescentic and mixed class.

Several studies found that immune complex or complement deposition in renal histopathology was associated with lower initial renal function, higher levels of proteinuria and poor renal outcome [24-26]. In the current study, only nine patients showed C3 deposition in glomerular and/or arteriolar walls. The eGFR at diagnosis, proteinuria and incidence of ESRD in these nine cases did not differ from those without C3, other immune complex or complement deposition.

\section{Immunohistochemical findings}

There was no significant difference regarding the development of ESRD between the crescentic and mixed class. Therefore we performed immunohistochemical staining on samples from 34 patients with crescentic or mixed class to examine whether immunohistochemical factors are useful to predict ESRD in ANCA-associated glomerulonephritis. The rates of WT1, CD68, and cytokeratin expression did not differ significantly between patients with or without ESRD (data not shown). However, Kaplan-Meier analysis demonstrated that higher $\alpha$-SMA immunoreactivity tended to be associated with poorer renal survival in crescentic and mixed class.

Several studies have provided evidence to suggest that glomerular component cells undergo mesenchymal transition in a pro-inflammatory environment. Epithelialmesenchymal transition (EMT) is widely accepted as a mechanism whereby injured epithelial and/or endothelial cells transform into mesenchymal cells, which contribute to the development of fibrosis $[27,28]$. EMT and myofibroblastic differentiation were originally thought to occur predominantly in the interstitium; however, several reports have shown that mesangial cells can also differentiate into myofibroblasts, resulting in fibrosis [28,29]. Barnes et al. [30] reported that myofibroblasts were mostly responsible for interstitial accumulation and consequent structural deformations associated with fibrosis. In renal disease, glomerular mesangial cells also acquire a myofibroblastic phenotype and synthesize the same matrix proteins. $\alpha$-SMA expression is considered to be a useful marker of myofibroblast differentiation in several disease settings [31-34]. We thought that a normal glomerulus under the light microscope might switch to a fibrogenic state if the glomerulus was positive for $\alpha$-SMA. Notably, glomeruli often appeared normal under light microscopy, but $\alpha$-SMA positive or negative glomeruli could be detected after careful observation. Consequently, $\alpha$-SMA-positive glomeruli may progress to fibrosis, glomerulosclerosis, and renal failure. However, $\alpha$-SMA positivity was not associated with renal survival. It could be the low power of the $\alpha$-SMA immunohistochemical analysis $(n=34)$, since the survival analysis did not reach significance but came close to it $(p=0.1074)$. Therefore, further studies are needed to clarify whether immunohistochemical staining of $\alpha$-SMA is a useful predictor for renal prognosis.

This study had several limitations. First, the sample size was relatively small, and this may have reduced the validity of the results. Second, no standard therapy was administered to all patients, and treatments were at the discretion of the attending physicians. Third, immunohistochemical analysis was not performed in all cases and we were therefore unable to perform Cox regression multivariate analysis of the relationship between $\alpha$-SMA 
positivity and ESRD. Fourth, the association between the histopathologic classification system and eGFR at 1 year was only based on an univariate analyses. Multivariate analysis with eGFR at 1 year as outcome was not performed. Finally, this current study was a retrospective study, thus further verification by a prospective study is desired.

\section{Conclusions}

The histopathological classification system proposed by Berden et al. [9] is a simple method, which is associated with eGFR at 1 year and tends to be associated with the incidence of ESRD in Japanese patients with ANCA-associated glomerulonephritis in our cohort. $\alpha$-SMA immunopositivity shows a trend toward an association with the renal survival rates of patients classified as crescentic or mixed class. Therefore, we suggest that immunohistochemical staining for $\alpha$-SMA expression, a widely used pathological method, might be useful to estimate renal outcome. Further verification of the histopathologic classification and $\alpha$-SMA staining for ANCA-associated glomerulonephritis is desired.

\section{Competing interests}

The authors declare that they have no competing interests.

\section{Authors' contributions}

TI, SF and KK made substantial contributions to conception and design. TI, SF, KK, JY, SU, YS, SH and TT were involved in acquisition and interpretation of data. TI, SF, YM and AY performed the statistical analysis and interpretation of data. TI, KK and AY performed histopathological evaluation SF, KF, TW, KK and AY advised throughout the study and its final approval and helped to draft the manuscript. All authors read and approved the final manuscript.

\section{Acknowledgements}

We thank Dr. Yuji Sato, Dr. Kazuhiro Yamada, Dr. Masao Kikuchi and Dr. Yasuyuki Shinozaki for acquisition and interpretation of data.

\section{Author details}

${ }^{1}$ Department of Pathology, University of Miyazaki, Miyazaki, Japan. ${ }^{2}$ Department of Hemovascular Medicine and Artificial Organs, University of Miyazaki, 5200 Kihara, Kiyotake, Miyazaki 889-1692, Japan. ${ }^{3}$ Division of Blood Purification, Kanazawa University Hospital, Kanazawa, Japan. ${ }^{4}$ Department of Internal Medicine, Toyama Prefectural Central Hospital, Toyama, Japan. ${ }^{5}$ Department of Internal Medicine, Miyazaki Prefectural Hospital, Miyazaki, Japan. ${ }^{6}$ Department of Pathology, Miyazaki Prefectural Hospital, Miyazaki, Japan. ${ }^{7}$ Department of Internal Medicine, Koga General Hospital, Miyazaki, Japan. ${ }^{8}$ Department of Internal Medicine, Miyazaki Social Insurance Konan Hospital, Miyazaki, Japan. ${ }^{9}$ Division of Nephrology, Department of Laboratory Medicine, Kanazawa University, Kanazawa, Japan. ${ }^{10}$ Division of Circulation and Body Fluid Regulation, Faculty of Medicine, University of Miyazaki, Miyazaki, Japan.

Received: 23 July 2012 Accepted: 12 June 2013

Published: 17 June 2013

\section{References}

1. Pettersson EE, Sundelin B, Heigl Z: Incidence and outcome of pauciimmune necrotizing and crescentic glomerulonephritis in adults. Clin Nephrol 1995, 43:141-149.

2. Jennette JC, Falk RJ: Small-vessel vasculitis. N Engl J Med 1997, 337:1512-1523.

3. Jennette JC, Falk RJ, Bacon PA, Basu N, Cid MC, Ferrario F, Flores-Suarez LF, Gross WL, Guillevin L, Hagen EC, Hoffman GS, Jayne DR, Kallenberg CG, Lamprecht P, Langford CA, Luqmani RA, Mahr AD, Matteson EL, Merkel PA, Ozen S, Pusey CD, Rasmussen N, Rees AJ, Scott DG, Specks U, Stone JH,
Takahashi K, Watts RA: 2012 revised International Chapel Hill Consensus Conference Nomenclature of Vasculitides. Arthritis Rheum 2013, 65:1-11.

4. Bajema IM, Hagen EC, Hermans J, Noël LH, Waldherr R, Ferrario F, Van Der Woude FJ, Bruijn JA: Kidney biopsy as a predictor for renal outcome in ANCA-associated necrotizing glomerulonephritis. Kidney Int 1999, 56:1751-1758.

5. Hauer HA, Bajema IM, Van Houwelingen HC, Ferrario F, Noël LH, Waldherr R, Jayne DR, Rasmussen N, Bruijn JA, Hagen EC: Determinants of outcome in ANCA-associated glomerulonephritis: A prospective clinico-histopathological analysis of 96 patients. Kidney Int 2002, 62:1732-1742.

6. Neumann I, Kain R, Regele H, Soleiman A, Kandutsch S, Meisl FT: Histological and clinical predictors of early and late renal outcome in ANCA-associated vasculitis. Nephrol Dial Transplant 2005, 20:96-104.

7. Day CJ, Howie AJ, Nightingale P, Shabir S, Adu D, Savage CO, Hewins P. Prediction of ESRD in pauci-immune necrotizing glomerulonephritis: quantitative histomorphometric assessment and serum creatinine. Am J Kidney Dis 2010, 55:250-258.

8. de Lind van Wijngaarden RA, Hauer HA, Wolterbeek R, Jayne DR, Gaskin G, Rasmussen N, Noël LH, Ferrario F, Waldherr R, Hagen EC, Bruijn JA, Bajema IM: Clinical and histologic determinants of renal outcome in ANCA-associated vasculitis: A prospective analysis of 100 patients with severe renal involvement. J Am Soc Nephrol 2006, 17:2264-2274.

9. Berden AE, Ferrario F, Hagen EC, Jayne DR, Jennette JC, Joh K, Neumann I, Noël LH, Pusey CD, Waldherr R, Bruijn JA, Bajema IM: Histopathologic classification of ANCA-associated glomerulonephritis. J Am Soc Nephrol 2010, 21:1628-1636.

10. Fujimoto S, Uezono S, Hisanaga S, Fukudome K, Kobayashi S, Suzuki K, Hashimoto $\mathrm{H}$, Nakao $\mathrm{H}$, Nunoi $\mathrm{H}$ : Incidence of ANCA-related primary renal vasculitisin the Miyazaki Prefecture: the first population-based, retrospective, epidemiologic survey in Japan. Clin J Am Soc Nephrol 2006 1:1016-1022.

11. Fujimoto S, Watts RA, Kobayashi S, Suzuki K, Jayne DR, Scott DG, Hashimoto H, Nunoi $\mathrm{H}$ : Comparison of the epidemiology of anti-neutrophil cytoplasmic antibody-associated vasculitis between Japan and the U.K. Rheumatology 2011, 50:1916-1920.

12. Watts R, Lane S, Hanslik T, Hauser T, Hellmich B, Koldingsnes W, Mahr A, Segelmark M, Cohen-Tervaert JW, Scott D: Development and validation of a consensus methodology for the classification of the ANCA-associated vasculitides and polyarteritis nodosa for epidemiological studies. Ann Rheum Dis 2007, 66:222-227.

13. Matsuo S, Imai E, Horio M, Yasuda Y, Tomita K, Nitta K, Yamagata K, Tomino Y, Yokoyama H, Hishida A: Revised equations for estimated GFR from serum creatinine in Japan. Am J Kidney Dis 2009, 53:982-992.

14. Bajema IM, Hagen EC, Hansen BE, Hermans J, Noël LH, Waldherr R, Ferrario F, van der Woude FJ, Bruijn JA: The renal histopathology in systemic vasculitis: An international survey study of inter- and intra-observer agreement. Nephrol Dial Transplant 1996, 11:1989-1995.

15. Shigematsu H, Yamaguchi N, Koyama A: Glomerulointerstitial events in rapidly progressive nephritic syndrome, with special reference to histologic grade and stage on the renal lesions. Clin Exp Nephrol 1998, 2:330-338.

16. Andrassy K, Erb A, Koderisch J, Waldherr R, Ritz E: Wegener's granulomatosis with renal involvement: patient survival and correlations between initial renal function, renal histology, therapy and renal outcome. Clin Nephrol 1991, 35:139-147.

17. Gans RO, Kuizinga MC, Goldschmeding R, Assmann K, Huysmans FT, Gerlag PG, Donker AJ, Hoorntje SJ: Clinical features and outcome in patients with glomerulonephritis and antineutrophil cytoplasmic autoantibodies. Nephron 1993, 64:182-188.

18. Hogan SL, Nachman PH, Wilkman AS, Jennette JC, Falk RJ: Prognostic markers in patients with antineutrophil cytoplasmic autoantibodyassociated microscopic polyangiitis and glomerulonephritis. J Am Soc Nephrol 1996, 7:23-32.

19. Franssen CF, Stegeman CA, Oost-Kort WW, Kallenberg CG, Limburg PC, Tiebosch A, De Jong PE, Tervaert JW: Determinants of renal outcome in anti-myeloperoxidase-associated necrotizing crescentic glomerulonephritis. J Am Soc Nephrol 1998, 9:1915-1923.

20. Hauer HA, Bajema IM, van Houwelingen HC, Ferrario F, Noël LH, Waldherr R, Jayne DR, Rasmussen N, Bruijn JA, Hagen EC, European Vasculitis Study Group (EUVAS): Renal histology in ANCA-associated vasculitis: differences between diagnostic and serologic subgroups. Kidney Int 2002, 61:80-89. 
21. Lapraik C, Watts R, Bacon P, Carruthers D, Chakravarty K, D'Cruz D, Guillevin L, Harper L, Jayne D, Luqmani R, Mooney J, Scott D: BSR and BHPR guidelines for the management of adults with ANCA associated vasculitis. Rheumatology 2007, 46:1615-1616.

22. Yamagata K, Usui J, Saito C, Yamaguchi N, Hirayama K, Mase K, Kobayashi M, Koyama A, Sugiyama H, Nitta K, Wada T, Muso E, Arimura Y, Makino H, Matsuo S: ANCA-associated systemic vasculitis in Japan: clinical features and prognostic changes. Clin Exp Nephrol 2012, 16:580-588.

23. Uezono S, Sato Y, Hara S, Hisanaga S, Fukudome K, Fujimoto S, Nakao H, Kitamura K, Kobayashi S, Suzuki K, Hashimoto H, Nunoi H: Outcome of ANCA-associated primary renal vasculitis in Miyazaki prefecture. Intern Med 2007, 46:815-822.

24. Haas M, Eustace JA: Immune complex deposits in ANCA-associated crescentic glomerulonephritis: a study of 126 cases. Kidney Int 2004, 65:2145-2152.

25. Chen $M$, Xing GQ, Yu F, Liu G, Zhao MH: Complement deposition in renal histopathology of patients with ANCA-associated pauci-immune glomerulonephritis. Nephrol Dial Transplant 2009, 24:1247-1252.

26. Gigante A, Salviani C, Giannakakis K, Rosato E, Barbano B, Moroso A, Gasperini ML, Nofroni I, Salsano F, Cianci R, Pugliese F: Clinical and histological outcome predictors in renal limited pauci-immune crescentic glomerulonephritis: a single centre experience. Int J Immunopathol Pharmacol 2012, 25:287-292.

27. Bariety J, Hill GS, Mandet C, Irinopoulou T, Jacquot C, Meyrier A, Bruneval P. Glomerular epithelial-mesenchymal transdifferentiation in pauci-immune crescentic glomerulonephritis. Nephrol Dial Transplant 2003, 18:1777-1784.

28. Iehara N, Takeoka H, Tsuji H, Imabayashi T, Foster DN, Strauch AR, Yamada Y, Kita T, Doi T: Differentiation of smooth muscle phenotypes in mouse mesangial cells. Kidney Int 1996, 49:1330-1341.

29. Schnaper HW, Hayashida T, Hubchak SC, Poncelet AC: TGF- $\beta$ signal transduction and mesangial cell fibrogenesis. Am J Physiol Renal Physiol 2003, 284:F243-F245.

30. Barnes $J \mathrm{~L}$, Gorin $Y$ : Myofibroblast differentiation during fibrosis: role of NAD(P)H oxidases. Kidney Int 2011, 79:944-956.

31. Johnson RJ, lida H, Alpers CE, Majesky MW, Schwartz SM, Pritzi P, Gordon K, Gown AM: Expression of smooth muscle cell phenotype by rat mesangial cells in immune complex nephritis. Alpha-smooth muscle actin is a marker of mesangial cell proliferation. J Clin Invest 1991, 87:847-858.

32. Desmouliere A, Darby IA, Gabbiani G: Normal and pathologic soft tissue remodeling: role of the myofibroblast, with special emphasis on liver and kidney fibrosis. Lab Invest 2003, 83:1689-1707.

33. Gabbiani G: The myofibroblast in wound healing and fibrocontractive diseases. J Pathol 2003, 200:500-503.

34. Kimura M, Asano M, Abe K, Miyazaki M, Suzuki T, Hishida A: Role of atrophic changes in proximal tubular cells in the peritubular deposition of type IV collagen in a rat renal ablation model. Nephrol Dial Transplant 2005, 20:1559-1565.

doi:10.1186/1471-2369-14-125

Cite this article as: Iwakiri et al: Validation of a newly proposed histopathological classification in Japanese patients with anti-neutrophil cytoplasmic antibody-associated glomerulonephritis. BMC Nephrology 2013 14:125.

\section{Submit your next manuscript to BioMed Central and take full advantage of:}

- Convenient online submission

- Thorough peer review

- No space constraints or color figure charges

- Immediate publication on acceptance

- Inclusion in PubMed, CAS, Scopus and Google Scholar

- Research which is freely available for redistribution 